\title{
Actuator-fault Detection and Isolation based on Interval Observers and Invariant Sets
}

\author{
Feng Xu, Vicenç Puig, Carlos Ocampo-Martinez, Florin Stoican, and Sorin Olaru
}

\begin{abstract}
This paper proposes an interval observer-based actuator fault detection and isolation (FDI) approach. An interval observer matching the healthy system mode is designed to monitor the system. When the system is in different modes, state or output interval vectors predicted by the interval observer manifest different dynamical behaviors. To guarantee reliable FDI, a collection of invariant set-based FDI conditions are established. Under these conditions, actuator faults can be accurately detected and isolated during the transition between different modes. At the end, the effectiveness of this proposed approach is presented by using a numerical example.
\end{abstract}

\section{INTRODUCTION}

Interval observer-based robust fault detection (FD) is the development of the well-known observer-based FD techniques [5]. Propagating the effect of uncertainties through the system models, an interval observer matching the current system mode can predict output intervals to bound real outputs. Generally, FD is performed by testing the consistency between predicted output intervals and real-time outputs.

In this approach, only the healthy interval observer is used to monitor the system. When the system is healthy, in steady state, the real outputs should be bounded by output intervals predicted by the interval observer. As fault occurrence always indicates system mode switching, the predicted output intervals generally have different dynamical behaviors under different faults. The behavioral difference of the interval observer under different faults provides the possibilities to isolate the faults, which is the fault isolation (FI) principle of the approach proposed in this paper.

The design of the interval observer is based on the Luenberger structure. For simplicity, only uncertainties and faults with known magnitudes are considered. Anyway, this approach can be extended to the case of parametric uncertainties and faults with unknown magnitudes but known bounds.

In the literature, only results concerning the interval observer-based FD can be found [3], while interval observerbased FI is still blank in the scientific community. Thus, this paper has two main contributions. First, it proposes a novel way to extend interval observers to FI applications. Second, the proposed approach ensures that the considered faults

F. Xu, V. Puig and C. Ocampo-Martinez are with the Institut de Robòtica i Informàtica Industrial (CSIC-UPC), Technical University of Catalonia (UPC), Llorens i Artigas, 4-6, 08028 Barcelona, Spain, \{fxu, vpuig, cocampo\}@iri.upc.edu.

F. Stoican is with the Dept. of Automatic Control and Systems Engineering, Faculty of Automatic Control and Computers, "Politehnica" University of Bucharest, 313 Spl. Independentei, 060042 Bucharest, Romania, florin.stoican@acse.pub.ro.

S. Olaru is with the E3S (SUPELEC Systems Sceinces), Automatic Control Departement, Gif sur Yvette, France, sorin.olaru@supelec.fr. are detectable and isolable during the transition between different modes as long as FDI conditions are satisfied.

Section II introduces zonotopes and invariant sets. Section III introduces the plant model and the interval observer. In Section IV, the expression of residual zonotopes and their bounding zonotopes are derived. The FDI algorithm is proposed in Section V. In Section VI, a collection of FDI conditions are established. In Section VII, a numerical example illustrates the effectiveness of the proposed approach. Section VIII draws some conclusions.

The notation $\oplus$ represents the Minkowski sum, |.| denotes the elementwise absolute value, $\mathbf{B}^{r}$ is a $r$-dimensional unitary box and the inequalities are explained elementwise.

\section{PRELIMINARIES}

\section{A. Zonotopes}

Definition 2.1: The interval hull $\square X$ of a zonotope ${ }^{1}$ $X=p \oplus G \mathbf{B}^{r} \subset \mathbb{R}^{n}$ is the smallest interval box that contains $X$, i.e., $\square X=\left\{x:\left|x_{i}-p_{i}\right| \leq\left\|G_{i}\right\|_{1}\right\}$, where $G_{i}$ is the $i$-th row of $G$, and $x_{i}$ and $p_{i}$ are the $i$-th components of $x$ and $p$, respectively.

Property 2.1: Given zonotopes $X_{1}=p_{1} \oplus G_{1} \mathbf{B}^{r_{1}} \subset \mathbb{R}^{n}$ and $X_{2}=p_{2} \oplus G_{2} \mathbf{B}^{r_{2}} \subset \mathbb{R}^{n}$, the Minkowski sum of them is $X_{1} \oplus X_{2}=\left\{p_{1}+p_{2}\right\} \oplus\left[\begin{array}{ll}G_{1} & G_{2}\end{array}\right] \mathbf{B}^{r_{1}+r_{2}}$.

Property 2.2: Given a zonotope $X=p \oplus G \mathbf{B}^{r} \subset \mathbb{R}^{n}$ and a compatible matrix $K, K X=K p \oplus K G \mathbf{B}^{r}$ holds.

Property 2.3: [2] Given a zonotope $X=p \oplus G \mathbf{B}^{r} \subset \mathbb{R}^{n}$ and an integer $s$ (with $n<s<r$ ), denote by $\hat{G}$ the matrix resulting from the recording of the columns of the matrix $G$ in decreasing Euclidean norm. $X \subseteq p \oplus\left[\hat{G}_{T} \quad Q\right] \mathbf{B}^{s}$ where $\hat{G}_{T}$ is obtained from the first $s-n$ columns of matrix $\hat{G}$ and $Q \in \mathbb{R}^{n \times n}$ is a diagonal matrix whose elements satisfy $Q_{i i}=\sum_{j=s-n+1}^{r}\left|\hat{G}_{i j}\right|, i=1, \ldots, n$.

Property 2.4: [1] Given a zonotope $X=p \oplus G \mathbf{B}^{r} \subset \mathbb{R}^{n}$, a strip denoted as $S=\left\{x \in \mathbb{R}^{n}|| c x-d \mid \leq \sigma\right\}$ and a vector denoted as $\lambda \in \mathbb{R}^{n}$, then $X \cap S \subseteq \hat{X}(\lambda)=\hat{p}(\lambda) \oplus \hat{G}(\lambda) \mathbf{B}^{r+1}$ where $\hat{p}(\lambda)=p+\lambda(d-c p)$ and $\hat{G}(\lambda)=\left[\begin{array}{ll}(I-\lambda c) G & \sigma \lambda\end{array}\right]$.

\section{B. Invariant Sets}

Theorem 2.1: [4] Considering the stable plant $x_{k+1}=$ $A_{\circ} x_{k}+B_{\circ} \delta_{k}$, where $A_{\circ}$ and $B_{\circ}$ are constant and $\delta_{k} \in \Delta=$ $\left\{\delta:\left|\delta-\delta^{\circ}\right| \leq \bar{\delta}\right\}$ with $\delta^{\circ}$ and $\bar{\delta}$ constant, and letting $A_{\circ}=V \Lambda V^{-1}$ be the Jordan decomposition of $A_{\circ}$,

$$
\begin{aligned}
\Phi(\theta)= & \left\{x \in \mathbb{R}^{n}:\left|V^{-1} x\right| \leq(I-|\Lambda|)^{-1}\left|V^{-1} B_{\circ}\right| \bar{\delta}\right. \\
& +\theta\} \oplus \xi^{\circ}
\end{aligned}
$$

\footnotetext{
${ }^{1}$ In this paper, $p$ and $G$ are called the center and segment matrix of the
} zonotope, respectively. 
is robust positively invariant (RPI) and attractive for the system trajectories, with $\theta$ any vector with positive components and $I$ the identity matrix.

1) For any $\theta$, the set $\Phi(\theta)$ is (positively) invariant, that is, if $x_{0} \in \Phi(\theta)$, then $x_{k} \in \Phi(\theta)$ for all $k \geq 0$.

2) Given $\theta \in \mathbb{R}^{n}, \theta>0$, and $x_{0} \in \mathbb{R}^{n}$, there exists $k^{*} \geq 0$ such that $x_{k} \in \Phi(\theta)$ for all $k \geq k^{*}$.

Proposition 2.1: [4] Considering the same plant and denoting $X_{0}$ as a RPI initial set, each of the set iterations

$$
X_{j+1}=A_{\circ} X_{j} \oplus B_{\circ} \Delta, \quad j \in \mathbb{N},
$$

where $j$ denotes the $j$-th element of the set sequence and $\mathbb{N}$ represents the set of natural numbers, is a RPI approximation of the minimal RPI (mRPI) set. Furthermore, as $j$ tends to infinity, the set sequence converges to the mRPI set.

\section{Plant Models And Interval ObSERVERS}

\section{A. Dynamical Models}

The linear discrete time-invariant model of the plant under actuator faults is considered as

$$
\begin{aligned}
x_{k+1} & =A x_{k}+B F_{i} u_{k}+\omega_{k}, \\
y_{k} & =C x_{k}+\eta_{k},
\end{aligned}
$$

where $A \in \mathbb{R}^{n \times n}, B \in \mathbb{R}^{n \times p}$ and $C \in \mathbb{R}^{q \times n}$ are constant matrices, $x_{k} \in \mathbb{R}^{n}, u_{k} \subset \mathbb{R}^{p}$ and $y_{k} \in \mathbb{R}^{q}$ are states, inputs and outputs at time instant $k$, respectively, $\omega_{k}$ and $\eta_{k}$ represent system uncertainties in states and outputs, respectively, and $F_{i}, i \in \mathbb{I}=\{0,1, \cdots, N\}$, is a $p \times p$ diagonal matrix modeling the $i$-th mode of the system, where $F_{0}$ is the identity matrix to describe the healthy mode ${ }^{2}$.

All diagonal elements of $F_{i}$ belong to $[0,1]$ where 0 and 1 represent the complete outage and health of the corresponding actuators, respectively, and a value in $(0,1)$ denotes partial performance degradation of actuators. It is assumed that $\omega_{k}$ and $\eta_{k}$ belong to

$$
\begin{aligned}
W & =\left\{\omega_{k} \in \mathbb{R}^{n}:\left|\omega_{k}-\omega^{c}\right| \leq \bar{\omega}, \omega^{c} \in \mathbb{R}^{n}, \bar{\omega} \in \mathbb{R}^{n}\right\}, \\
V & =\left\{\eta_{k} \in \mathbb{R}^{q}:\left|\eta_{k}-\eta^{c}\right| \leq \bar{\eta}, \eta^{c} \in \mathbb{R}^{q}, \bar{\eta} \in \mathbb{R}^{q}\right\}
\end{aligned}
$$

where $\omega^{c}, \eta^{c}, \bar{\omega}$ and $\bar{\eta}$ are constant vectors. Furthermore, $W$ and $V$ can be rewritten as zonotopes

$$
\begin{gathered}
W=\omega^{c} \oplus H_{\bar{\omega}} \mathbf{B}^{n}, \\
V=\eta^{c} \oplus H_{\bar{\eta}} \mathbf{B}^{q},
\end{gathered}
$$

where $H_{\bar{\omega}} \in \mathbb{R}^{n \times n}$ and $H_{\bar{\eta}} \in \mathbb{R}^{q \times q}$ are diagonal matrices with the main diagonal being $\bar{\omega}$ and $\bar{\eta}$, respectively.

Assumption 3.1: The plant is stable and the pair $(A, C)$ is detectable.

Assumption 3.2: One and only one persistent fault occurs at one time and the system keeps operating in a mode such that it goes into steady state of the mode before a mode switching induced by a fault.

\footnotetext{
${ }^{2} \mathbb{I}$ represents a finite range of actuator fault modes more critical to the system.
}

\section{B. Interval Observers}

According to the healthy system model (1), the healthy interval observer is designed as

$$
\begin{aligned}
\hat{X}_{k+1}= & (A-L C) \hat{X}_{k} \oplus\left\{B F_{0} u_{k}\right\} \oplus\left\{L y_{k}\right\} \\
& \oplus(-L) V \oplus W, \\
\hat{Y}_{k}= & C \hat{X}_{k} \oplus V,
\end{aligned}
$$

where $\hat{X}_{k}$ and $\hat{Y}_{k}$ are predicted state and output zonotopes, and $L$ is chosen to ensure the observer contractiveness.

Assumption 3.3: The initial plant state is denoted as $x_{0}$ and the initial zonotope for the interval observer is denoted as $\hat{X}_{0}$ and $x_{0} \in \hat{X}_{0}$ holds.

According to (2), Property 2.1 and Property 2.2, the center $\hat{x}_{k+1}^{c}$ and segment matrix $\hat{H}_{k+1}^{x}$ of $\hat{X}_{k+1}$, and the center $\hat{y}_{k}^{c}$ and segment matrix $\hat{H}_{k}^{y}$ of $\hat{Y}_{k}$ are computed as

$$
\begin{aligned}
\hat{x}_{k+1}^{c} & =(A-L C) \hat{x}_{k}^{c}+B F_{0} u_{k}+L y_{k}-L \eta^{c}+w^{c}, \\
\hat{H}_{k+1}^{x} & =\left[\begin{array}{lll}
(A-L C) \hat{H}_{k}^{x} & -L H_{\bar{\eta}} & H_{\bar{\omega}}
\end{array}\right], \\
\hat{y}_{k}^{c} & =C \hat{x}_{k}^{c}+\eta^{c}, \\
\hat{H}_{k}^{y} & =\left[\begin{array}{ll}
C \hat{H}_{k}^{x} & H_{\bar{\eta}}
\end{array}\right] .
\end{aligned}
$$

As $k$ increases, the order of predicted zonotopes grows up dramatically. Thus, Property 2.3 is used to reduce the order of predicted state and output zonotopes.

\section{FDI using Interval Observers}

According to [3], the interval observer-based FD consists in testing whether

$$
\mathbf{0} \in \square R_{k},
$$

where 0 represents the zero vector and $R_{k}=\left\{y_{k}\right\} \oplus\left(-\hat{Y}_{k}\right)$ is the predicted residual zonotope at time instant $k$.

The proposed FI principe is that, when the system is healthy, predicted residual zonotopes always contain $\mathbf{0}$ while the predicted residual zonotopes will exclude $\mathbf{0}$ and vanish to a different domain determined by the particular faulty mode once the system is affected by a fault.

The prediction of interval observers and the computation of interval vectors are based on zonotopes and their interval hulls, respectively. For simplicity, all the following discussions are directly based on zonotopes.

\section{RESiduAl Zonotopes And Bounding Zonotopes}

\section{A. Residual Zonotopes}

Whenever a fault occurs, residual zonotopes start to drift towards different domains which are no longer centered at $\mathbf{0}$ because of the model mismatch between the system current model and the interval observer internal model.

According to (1) and (2), the expression of residual zonotopes $^{3}$ is further derived as

$$
\begin{aligned}
R_{k} & =\left\{y_{k}\right\} \oplus\left(-\hat{Y}_{k}\right) \\
& =C\left\{\left\{x_{k}\right\} \oplus\left(-\hat{X}_{k}\right)\right\} \oplus\left\{\eta_{k}\right\} \oplus(-V) .
\end{aligned}
$$

\footnotetext{
${ }^{3} R_{k}$ generally represents residual zonotopes predicted by the interval observer whereas $R_{k}^{i}$ is especially used to denote predicted residual zonotopes under the $i$-th mode.
} 
To obtain the residual zonotopes, those zonotopes defined as $\tilde{X}_{k}=\left\{x_{k}\right\} \oplus\left(-\hat{X}_{k}\right)$ should be firstly considered. When the system is healthy, $\tilde{X}_{k}$ is written as

$$
\begin{aligned}
\tilde{X}_{k}^{0} & =\left\{x_{k}\right\} \oplus\left(-\hat{X}_{k}\right) \\
& =\left\{x_{k}-\hat{x}_{k}^{c}\right\} \oplus \hat{H}_{k}^{x} \mathbf{B}^{s_{k}} .
\end{aligned}
$$

According to (1), (2) and (3), and using $\tilde{x}_{k}^{0, c}$ and $\tilde{H}_{k}^{0, x}$ to denote $x_{k}-\hat{x}_{k}^{c}$ and $\hat{H}_{k}^{x}$ in (6), respectively, the center and segment matrix of $\tilde{X}_{k+1}^{0}$ can be computed as

$$
\begin{aligned}
\tilde{x}_{k+1}^{0, c} & =(A-L C) \tilde{x}_{k}^{0, c}-L\left(\eta_{k}-\eta^{c}\right)+\left(\omega_{k}-\omega^{c}\right), \\
\tilde{H}_{k+1}^{0, x} & =\hat{H}_{k+1}^{x}=\left[(A-L C) \hat{H}_{k}^{x}-L H_{\bar{\eta}} \quad H_{\bar{\omega}}\right] .
\end{aligned}
$$

Similarly, when the system is in the $i$-th $(i \in \mathbb{I} \backslash\{0\})$ faulty mode, the zonotope $\tilde{X}_{k}$ is written as $\tilde{X}_{k}^{i}$ with the same expression as in (6). According to (1), (2) and (3), and using $\tilde{x}_{k}^{i, c}$ and $\tilde{H}_{k}^{i, x}$ to replace $x_{k}-\hat{x}_{k}^{c}$ and $\hat{H}_{k}^{x}$ in (6), respectively, the center and segment matrix of $\tilde{X}_{k+1}^{i}$ are computed as

$$
\begin{aligned}
\tilde{x}_{k+1}^{i, c}= & (A-L C) \tilde{x}_{k}^{i, c}+B\left(F_{i}-F_{0}\right) u_{k}-L\left(\eta_{k}-\eta^{c}\right) \\
& +\left(\omega_{k}-\omega^{c}\right), \\
\tilde{H}_{k+1}^{i, x}= & \hat{H}_{k+1}^{x}=\left[\begin{array}{lll}
(A-L C) \hat{H}_{k}^{x} & -L H_{\bar{\eta}} & H_{\bar{\omega}}
\end{array}\right] .
\end{aligned}
$$

Eventually, according to (5), the residual zonotopes in the healthy mode and the $i$-th faulty mode are deduced as

$$
\begin{aligned}
& R_{k}^{0}=C \tilde{X}_{k}^{0} \oplus\left\{\eta_{k}\right\} \oplus(-V), \\
& R_{k}^{i}=C \tilde{X}_{k}^{i} \oplus\left\{\eta_{k}\right\} \oplus(-V) .
\end{aligned}
$$

According to Definition 2.1, in this paper, the interval hull width of a zonotope is defined as follows.

Definition 4.1: The interval hull width ${ }^{4}$ of a zonotope $X=p \oplus G \mathbf{B}^{r} \subset \mathbb{R}^{n}$ is defined as follows: width $(X)=(2 \|$ $\left.G_{1}\left\|_{1}, 2\right\| G_{2}\left\|_{1}, \cdots, 2\right\| G_{n} \|_{1}\right)$, where $G_{i}(1 \leq i \leq n)$ is the $i$-th row of $G$.

Remark 1: According to (7b) and (8b), the segment matrix expression of $\tilde{X}_{k}^{0}$ is the same as that of $\tilde{X}_{k}^{i}$, which means that the mode switching does not affect the interval hull width of $R_{k}$ and no matter which mode the system is in, $\operatorname{width}\left(R_{\infty}\right)$ is constant.

Practically, one uses the interval hull width of a residual zonotope $R_{k_{a}}$ at time instant $k_{a}$ to approximate $\operatorname{width}\left(R_{\infty}\right)$, as long as the approximation is sufficiently precise.

\section{B. Residual-bounding Zonotopes}

This subsection establishes bounding zonotopes for (7) and (8) by using the zonotopes of uncertainties.

A zonotope to bound $\tilde{X}_{k+1}^{0}$ is denoted as $\check{X}_{k+1}^{0}=\check{x}_{k+1}^{0, c} \oplus$ $\check{H}_{k+1}^{0, x} \mathbf{B}^{\check{s}_{k+1}^{0}}$. Using $W$ and $V$ to replace $\omega_{k}$ and $\eta_{k}$ in (7a), the center and segment matrix of $\check{X}_{k+1}^{0}$ are derived as

$$
\begin{aligned}
\check{x}_{k+1}^{0, c} & =(A-L C) \check{x}_{k}^{0, c}, \\
\check{H}_{k+1}^{0, x} & =\left[\begin{array}{lllll}
(A-L C) \hat{H}_{k}^{x} & -L H_{\bar{\eta}} & L H_{\bar{\eta}} & H_{\bar{\omega}} & -H_{\bar{\omega}}
\end{array}\right] .
\end{aligned}
$$

\footnotetext{
${ }^{4}$ If $X$ is an empty set, $\operatorname{width}(X)=\mathbf{0}$.
}

The zonotope-based dynamics of (10) is established as

$$
\check{X}_{k+1}^{0}=(A-L C) \check{X}_{k}^{0} \oplus L(-V) \oplus W \oplus L V \oplus(-W) .
$$

Similarly, a bounding zonotope for $\tilde{X}_{k+1}^{i}$ in (8) is denoted as $\check{X}_{k+1}^{i}=\check{x}_{k+1}^{i, c} \oplus \check{H}_{k+1}^{i, x} \mathbf{B}^{\check{s}_{k+1}^{i}}$. The center and segment matrix of $\check{X}_{k+1}^{i}$ are derived as

$$
\begin{aligned}
\check{x}_{k+1}^{i, c} & =(A-L C) \check{x}_{k}^{i, c}+B\left(F_{i}-F_{0}\right) u_{k}, \\
\check{H}_{k+1}^{i, x} & =\left[\begin{array}{llll}
(A-L C) \hat{H}_{k}^{x} & -L H_{\bar{\eta}} & L H_{\bar{\eta}} & H_{\bar{\omega}}-H_{\bar{\omega}}
\end{array}\right],
\end{aligned}
$$

and a set-based dynamics of (12) is given as

$$
\begin{aligned}
\check{X}_{k+1}^{i}= & (A-L C) \check{X}_{k}^{i} \oplus B\left(F_{i}-F_{0}\right)\left\{u_{k}\right\} \oplus L(-V) \oplus W \\
& \oplus L V \oplus(-W) .
\end{aligned}
$$

Remark 2: It is known that, for all $k^{*} \geq k$, zonotopes predicted by (7) and (8) are always bounded by those predicted by (11) and (13), respectively, i.e., $\tilde{X}_{k^{*}}^{0} \subseteq \check{X}_{k^{*}}^{0}$ and $\tilde{X}_{k^{*}}^{i} \subseteq \check{X}_{k^{*}}^{i}$, as long as $\tilde{X}_{k}^{0} \subseteq \check{X}_{k}^{0}$ and $\tilde{X}_{k}^{i} \subseteq \check{X}_{k}^{i}$ hold.

Furthermore, according to (5), residual-bounding zonotopes of $R_{k}^{0}$ and $R_{k}^{i}$ can be respectively computed as

$$
\begin{aligned}
\check{R}_{k}^{0} & =C \check{X}_{k}^{0} \oplus V \oplus(-V), \\
\check{R}_{k}^{i} & =C \check{X}_{k}^{i} \oplus V \oplus(-V) .
\end{aligned}
$$

Note that, as long as Remark 2 is satisfied, the persistent inclusion $R_{k}^{0} \subseteq \check{R}_{k}^{0}$ and $R_{k}^{i} \subseteq \check{R}_{k}^{i}$ always hold.

\section{FDI AND System STATUS DETERMination ALGORITHM}

\section{A. Fault Detection and Isolation}

It is assumed that the system is firstly healthy. Thus, the residual zonotopes $R_{k}$ always include $\mathbf{0}$. FD consists in realtime testing whether the criterion (4) is violated.

Furthermore, it is assumed that a fault is detected at time instant $k_{d}$, thus, $\hat{X}_{k_{d}}$ and $R_{k_{d}}$ can be obtained. At $k_{d}$, one can construct an initial zonotope $\check{X}_{k_{d}}$ to initialize all faulty bounding zonotopes dynamics $\check{X}_{k}^{i}(i=\mathbb{I} \backslash\{0\})$, such that all the $\tilde{X}_{k_{d}}^{i}$ contains $\tilde{X}_{k_{d}}$, i.e., $\check{R}_{k_{d}}^{i}$ contains $R_{k_{d}}$.

After initializing, residual-bounding zonotopes matching the current system mode always bound $R_{k}$ after $k_{d}$ whereas the rest of residual-bounding zonotopes not matching the current system mode can only bound $R_{k}$ at the first several time instants after $k_{d}$ and finally diverge from $R_{k}$.

Thus, starting from the fault-detected time instant $k_{d}$, the fault can be isolated by real-time testing if

$$
R_{l_{d}} \subseteq \check{R}_{l_{d}}^{i}, \quad i=1,2, \ldots, N, l_{d}>k_{d},
$$

is violated for all the $\check{R}_{k}^{i}$ at each time instant, respectively, where $i$ represents the $i$-th faulty mode.

By repeatedly testing (15) at each time instant after $k_{d}$, the time instant when there is one and only one residualbounding zonotope that can contain the current residual zonotope $R_{k}$ is the fault-isolated time instant. Under a set of conditions established in next sections, this FI approach can isolate faults during the transition induced by a fault. 


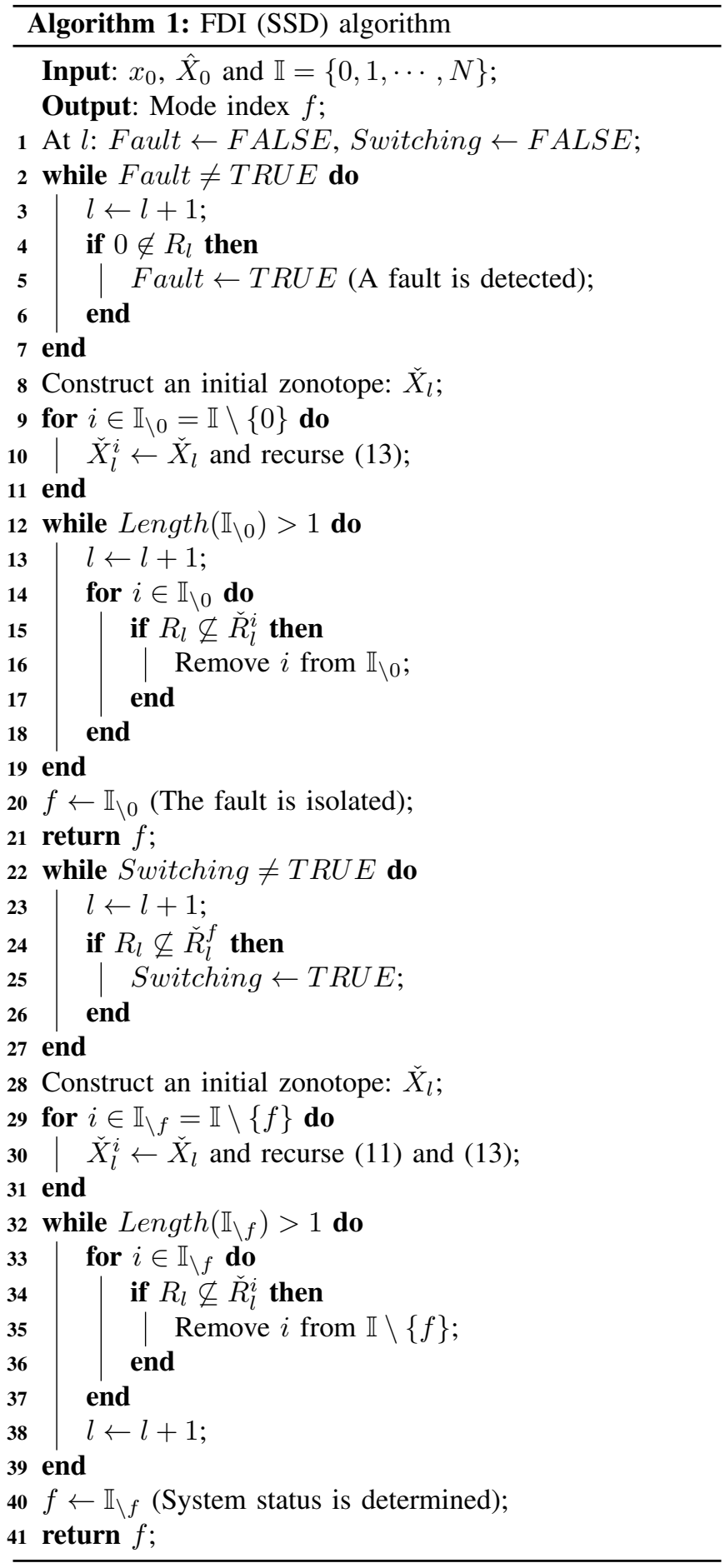

\section{B. System Status Determination (SSD)}

It is assumed that a fault is isolated at time instant $k_{i}$ and this fault corresponds to the $m$-th mode. In this mode, the residual zonotopes $R_{k}\left(k>k_{i}\right)$ should be bounded by the $m$-th residual-bounding zonotopes $\check{R}_{k}^{m}$. Thus, a mode switching from the $m$-th one can be detected if

$$
R_{k} \subseteq \check{R}_{k}^{m}, k>k_{i}
$$

is violated at time instant $k$. Otherwise, it is still considered that the system is in the $m$-th mode.

Furthermore, it is assumed that a mode switching is detected at $l_{i}$. Similarly, by using an initial zonotope to initialize the dynamics of $\check{X}_{k}^{0}$ and all $\check{X}_{k}^{i}(i \neq 0, m)$ such that $\check{R}_{l_{i}}^{0}$ and all $\check{R}_{l_{i}}^{i}(i \neq 0, m)$ contain $R_{l_{i}}$, then SSD can be identified by finding the residual-bounding zonotope $\check{R}_{k}^{i}$ $(i=\mathbb{I} \backslash\{m\})$ that can persistently bound $R_{k}$ for $k \geq l_{i}$.

Finally, the index of the found residual-bounding zonotope indicates the new system mode. Algorithm 1 collects the FDI and SSD procedures, where the operator Length(.) computes the number of elements in a set.

\section{Initial Zonotope for $\check{X}_{k}^{i}$}

It is assumed that the system is in the $m$-th mode, a fault is detected at time instant $k_{d}$. According to Section $\mathrm{V}-\mathrm{A}$ and $\mathrm{V}-\mathrm{B}$, the aforementioned initialization at $k_{d}$ is a key precondition for the effectiveness of the proposed FDI approach. Thus, a crucial point is to construct an initial zonotope $\check{X}_{k_{d}}$ at $k_{d}$ for all the corresponding bounding zonotopes $\check{X}_{k}^{i}(i=\mathbb{I} \backslash\{m\})$.

Comparing (7) and (8) with (10) and (12), respectively, it is known that $\tilde{X}_{k_{d}}^{u}$ can satisfy the requirement as the initial zonotope at $k_{d}$, where $u$ denotes the index of the current new and unknown mode. Thus, a zonotope that bounds $\tilde{X}_{k_{d}}^{u}$ can also be used as an initial zonotope. But, since $\tilde{X}_{k_{d}}^{u}$ is unknown, instead, the idea is to use the obtainable information $R_{k_{d}}$ to construct an initial zonotope $\check{X}_{k_{d}}$.

Defining $V_{0}=H_{\bar{\eta}} \mathbf{B}^{q}$, at $k_{d}$, (5) can be transformed into

$$
R_{k_{d}}=C\left\{\left\{x_{k_{d}}\right\} \oplus\left(-\hat{X}_{k_{d}}\right)\right\} \oplus\left\{\eta_{k_{d}}-\eta^{c}\right\} \oplus\left(-V_{0}\right) \text {. }
$$

By adding $-\left(\eta_{k_{d}}-\eta^{c}\right)$ to both sides of (17), (17) turns into

$$
R_{k_{d}} \oplus\left\{-\left(\eta_{k_{d}}-\eta^{c}\right)\right\}=C\left\{\left\{x_{k_{d}}\right\} \oplus\left(-\hat{X}_{k_{d}}\right)\right\} \oplus\left(-V_{0}\right),
$$

and considering $-\left(\eta_{k_{d}}-\eta^{c}\right) \in\left(-V_{0}\right)$, one has

$$
C\left\{\left\{x_{k_{d}}\right\} \oplus\left(-\hat{X}_{k_{d}}\right)\right\} \oplus\left(-V_{0}\right) \subseteq R_{k_{d}} \oplus\left(-V_{0}\right) .
$$

Thus, a key condition $C\left\{\left\{x_{k_{d}}\right\} \oplus\left(-\hat{X}_{k_{d}}\right)\right\} \subseteq R_{k_{d}}$ is obtained, i.e.,

$$
C \tilde{X}_{k_{d}}^{u} \subseteq R_{k_{d}} .
$$

$R_{k_{d}}$ is denoted as $R_{k_{d}}=r_{k_{d}}^{c} \oplus H_{k_{d}}^{r} \mathbf{B}^{s_{k_{d}}^{r}}, C_{i}$ and $H_{k_{d i}}^{r}$ denotes the $i$-th row of $C$ and $H_{k_{d}}^{r}$, respectively, and $r_{k_{d i}}^{c}$ is the $i$-th component of $r_{k_{d}}^{c}$. According to (18), one has

$$
\left|C_{i} \tilde{x}_{k_{d}}-r_{k_{d i}}^{c}\right| \leq\left\|H_{k_{d i}}^{r}\right\|_{1}, i=1,2, \cdots, q .
$$

It is seen that (19) includes $q$ equations, each of which determines a strip. If the intersection of all the $q$ strips is a closed set, denoted as $\tilde{\tilde{X}}_{k_{d}}$, then $\tilde{\tilde{X}}_{k_{d}}$ can bound $\tilde{X}_{k_{d}}^{u}$.

Assumption 5.1: The output matrix $C$ ensures that the intersection of (19) is closed. $\tilde{X}$ is a given zonotope determined by the physical constraints of the plant and $\tilde{X}$ can always be chosen to bound $\tilde{X}_{k}^{i}$ for $k \geq 0, i \in \mathbb{I}$.

Thus, an initial zonotope $\check{X}_{k_{d}}$ can be found by computing a zonotope overapproximation $\bar{X}_{k}$ for $\tilde{\tilde{X}}_{k}$ and this overapproximation can be computed according to Property 2.4. 
Property 2.4 computes zonotope approximations for the intersection of a zonotope and a strip. In order to compute $\bar{X}_{k}$, an initial zonotope is required by Property 2.4 and $\tilde{X}$ in Assumption 5.1 is used as the initial zonotope.

Thus, at $k_{d}$, by letting $\check{X}_{k_{d}}=\bar{X}_{k_{d}}$ to initialize all the corresponding bounding zonotopes in (11) and (13), FI (SSD) during the transition can be implemented.

In the case that $C$ is invertible, (18) is transformed as

$$
\tilde{X}_{k_{d}}^{u} \subseteq C^{-1} R_{k_{d}}
$$

where $C^{-1}$ is the inverse of $C$. In this case, $C^{-1} R_{k_{d}}$ is directly used as an initial zonotope, i.e., $\check{X}_{k_{d}}=C^{-1} R_{k_{d}}$.

\section{FDI AND SSD CONDITIONS}

\section{A. Bounding Zonotopes for $\check{X}_{k}^{i}$}

When the system is healthy, a zonotope to bound $\check{X}_{k+1}^{0}$ in (10) is denoted as $\breve{X}_{k+1}^{0}=\breve{x}_{k+1}^{0, c} \oplus \breve{H}_{k+1}^{0, x} \mathbf{B}^{\breve{s}_{k+1}^{0}}$. For simplicity, $\check{X}_{k+1}^{0}$ can be directly defined as

$$
\breve{X}_{k+1}^{0}=\check{X}_{k+1}^{0}
$$

To establish guaranteed FDI conditions, it is assumed that all possible values of control inputs $u_{k}$ belong to

$$
U=\left\{u_{k} \in \mathbb{R}^{p}:\left|u_{k}-u^{c}\right| \leq \bar{u}, u^{c} \in \mathbb{R}^{p}, \bar{u} \in \mathbb{R}^{p}\right\},
$$

where $u^{c}$ and $\bar{u}$ are constant. Further, $U$ can be rewritten as a zonotope $U=u^{c} \oplus H_{\bar{u}} \mathbf{B}^{p}$, where $H_{\bar{u}} \in \mathbb{R}^{p \times p}$ is a diagonal matrix with the diagonal entries composed of $\bar{u}$.

Similarly, a zonotope to bound $\check{X}_{k+1}^{i}$ in (12) is denoted as $\breve{X}_{k+1}^{i}=\breve{x}_{k+1}^{i c} \oplus \breve{H}_{k+1}^{i x} \mathbf{B}^{\breve{s}_{k+1}^{i}}$. Using $U$ to replace $u_{k}$ in (12a), the center and segment matrix of $\breve{X}_{k+1}^{i}$ are derived as

$$
\begin{aligned}
& \breve{x}_{k+1}^{i c}=(A-L C) \breve{x}_{k}^{i c}+B\left(F_{i}-F_{0}\right) u^{c}, \\
& \breve{H}_{k+1}^{i x}=\left[\begin{array}{lll}
(A-L C) \hat{H}_{k}^{x} & B\left(F_{i}-F_{0}\right) H_{u} & -L H_{\bar{\eta}} \\
L H_{\bar{\eta}} & H_{\bar{\omega}} & -H_{\bar{\omega}}
\end{array}\right] .
\end{aligned}
$$

An equivalent set-based dynamics for (21) is derived as

$$
\begin{aligned}
\breve{X}_{k+1}^{i}= & (A-L C) \breve{X}_{k}^{i} \oplus B\left(F_{i}-F_{0}\right) U \oplus L(-V) \oplus W \\
& \oplus L V \oplus(-W) .
\end{aligned}
$$

As per Theorem 2.1, one computes the corresponding invariant sets for $\breve{X}_{\infty}^{0}$ and $\breve{X}_{\infty}^{i}$, respectively. Using these invariant sets as initial sets of (11) and (22), $\breve{X}_{\infty}^{0}$ and $\breve{X}_{\infty}^{i}$ can be obtained by infinitely iterating (11) and (22), respectively.

It is impossible to compute sets at infinity. Instead, one can only obtain $R P I$ approximations $\breve{S}_{0}$ and $\breve{S}_{i}$ for $\breve{X}_{\infty}^{0}$ and $\breve{X}_{\infty}^{i}$, respectively. As long as $\breve{S}_{0}$ and $\breve{S}_{i}$ are sufficiently precise, they can be used to replace $\breve{X}_{\infty}^{0}$ and $\breve{X}_{\infty}^{i}$.

Remark 3: The best is that $\breve{S}_{0}$ and $\breve{S}_{i}$ are RPI approximations of the limit sets $\breve{X}_{\infty}^{0}$ and $\breve{X}_{\infty}^{i}$. But, in case that $\breve{S}_{0}$ and $\breve{S}_{i}$ are not RPI approximations, they are still acceptable as long as they sufficiently approximate $\breve{X}_{\infty}^{0}$ and $\breve{X}_{\infty}^{i}$.

\section{B. Guaranteed FDI Conditions}

As per (11), (14), (20) and (22), bounding zonotopes $\breve{R}_{k}^{0}$ and $\breve{R}_{k}^{i}$ of $\check{R}_{k}^{0}$ and $\check{R}_{k}^{i}$ are derived as

$$
\begin{aligned}
\breve{R}_{k}^{0} & =C \breve{X}_{k}^{0} \oplus V \oplus(-V), \\
\breve{R}_{k}^{i} & =C \breve{X}_{k}^{i} \oplus V \oplus(-V) .
\end{aligned}
$$

Theorem 6.1: Considering the plant (1), the interval observer (2), actuator modes II, Assumption 3.1, 3.2, 3.3 and 5.1 , and the boundedness of uncertainties, once a fault occurs, the detection and isolation of the fault can be guaranteed as long as the FDI conditions hold:

1) FD conditions:

$$
\mathbf{0} \in \breve{R}_{\infty}^{0} \text { and } \mathbf{0} \notin \breve{R}_{\infty}^{i}, \quad i \in \mathbb{I} \backslash 0,
$$

2) FI conditions:

width $\left(R_{\infty}\right) \not \leq$ width $\left(\breve{R}_{\infty}^{i} \cap \breve{R}_{\infty}^{j}\right), i \neq j, i, j \in \mathbb{I}$. (25)

Proof : 1) The condition (24) means that, in the faulty modes, residual zonotopes can not contain the zero vector, which assures that all considered faults can be detected.

2) According to Remark 1, the mode switching only affects the center of residual zonotopes and does not affect the interval hull width. If (25) holds, it implies that at infinity one and only one residual-bounding zonotope that can fully include $R_{\infty}$, which indicates the fault.

Since the transition is determined by the eigenvalues of $A-L C$ in (2), (11) and (22), once the transition completely disappears, the dynamical behaviors of (11) and (22) enter into steady state. Thus, the steady state behaviors approximate the system behaviors at infinity. Thus, (25) can ensure that all residual-bounding zonotopes not matching the current system mode will finally diverge from $R_{k}$.

Since it is impossible to compute zonotopes at infinity, the FDI conditions (24) and (25) only have theoretical value. In practice, one always turns to approximations. According to Section VI-A, $\breve{R}_{\infty}^{i}$ can be approximated by

$$
\stackrel{\circ}{R}_{\infty}^{i}=C \breve{S}_{i} \oplus V \oplus(-V), \quad i \in \mathbb{I} .
$$

Thus, the off-line checking of the guaranteed FDI conditions is to use the approximations in (26) to replace the corresponding sets in (24) and (25).

Note that, although the analysis of the proposed approach is based on zonotopes, the predictions of the interval observer and the prechecking of the FDI conditions are based on the interval hull of zonotopes.

\section{NumericAl EXAMPLE}

The numerical example only considers two different actuator faults $F_{1}$ and $F_{2}$ and the dynamics of the example is described as (1). The healthy interval observer is designed as (2) and the residual zonotopes are defined as (5). The parameters of the illustrative example are given as

$$
\begin{aligned}
& \text { - model parameters: } A=\left[\begin{array}{cc}
0.8667 & -1.2343 \\
0.01 & 1
\end{array}\right], \\
& B=\left[\begin{array}{cc}
0.01 & 1 \\
1 & 0.01
\end{array}\right], C=\left[\begin{array}{cc}
0.5 & 0 \\
0 & 1.5
\end{array}\right],
\end{aligned}
$$


- disturbances: $\bar{w}=\left[\begin{array}{ll}0 & 0\end{array}\right]^{T}, w^{c}=\left[\begin{array}{ll}0 & 0\end{array}\right]^{T}$,

- noises: $\bar{\eta}=\left[\begin{array}{ll}0.05 & 0.05\end{array}\right]^{T}, \eta^{c}=\left[\begin{array}{ll}0.15 & 0.15\end{array}\right]^{T}$,

- observer gain: $L=\left[\begin{array}{cc}0.3334 & -0.8229 \\ 0.02 & 0.1333\end{array}\right]$,

- faults: $F_{1}=\left[\begin{array}{cc}0.75 & 0 \\ 0 & 0.75\end{array}\right], F_{2}=\left[\begin{array}{cc}0.9 & 0 \\ 0 & 0.9\end{array}\right]$,

- sinusoidal inputs: $u^{c}=\left[\begin{array}{l}2 \\ 2\end{array}\right], H_{\bar{u}}=\left[\begin{array}{cc}0.2 & 0 \\ 0 & 0.2\end{array}\right]$,

- $x_{0}=\left[\begin{array}{l}0 \\ 0\end{array}\right], \hat{X}_{0}=\left[\begin{array}{l}0.1 \\ 0.1\end{array}\right] \oplus\left[\begin{array}{ccc}0.5 & 0 & 0 \\ 0 & 0 & 0.5\end{array}\right] \mathbf{B}^{3}$.

After iterating (7b), (11) and (22) fifty steps offline, width $\left(R_{k}\right), \breve{R}_{k}^{0}, \breve{R}_{k}^{1}$ and $\breve{R}_{k}^{2}$ are almost constant. Thus, these fifty-step iterations can be used as the approximations for width $\left(R_{\infty}\right), \breve{R}_{\infty}^{0}, \breve{R}_{\infty}^{1}$ and $\breve{R}_{\infty}^{2}$ to check the FDI conditions. The two components of the approximations are listed as

- $\operatorname{width}\left(R_{50}\right)=(0.1816,0.2150)$,

- $\square \stackrel{\circ}{R}_{50}^{0}=([-0.1816,0.1816],[-0.2150,0.2150])$,

- $\square \stackrel{\circ}{R}^{1}=([-1.1074,-0.5759],[-4.3812,-3.1937])$,

- $\square R_{50}^{2}=([-0.5519,-0.1214],[-1.8815,-1.1485])$,

- $\operatorname{width}\left(\square R_{50}^{0} \cap \square \stackrel{\circ}{R 0}_{50}^{1}\right)=(0,0)$,

- $w i d t h\left(\square R_{50}^{0} \cap \square \stackrel{R}{R}^{2}\right)=(0.0602,0)$,

- $\operatorname{width}\left(\square \dot{R}_{50}^{0} \cap \square \stackrel{\circ}{R}_{50}^{1}\right)=(0,0)$.

It is seen that the example satisfies (24) and (25) and the proposed approach can be used for FDI. FDI of the two faults is separately simulated. The scenarios for both faults are set as follows: from time instant 0 to 50 the system is healthy, from time instant 51 to 100 a fault occurs, from time instant 101 to 150 the system gets healthy again.

FDI is illustrated in Figures 1 and 2, which shows that the faults can be firstly detected and then isolated during the transition and is consistent with the theoretical analysis.
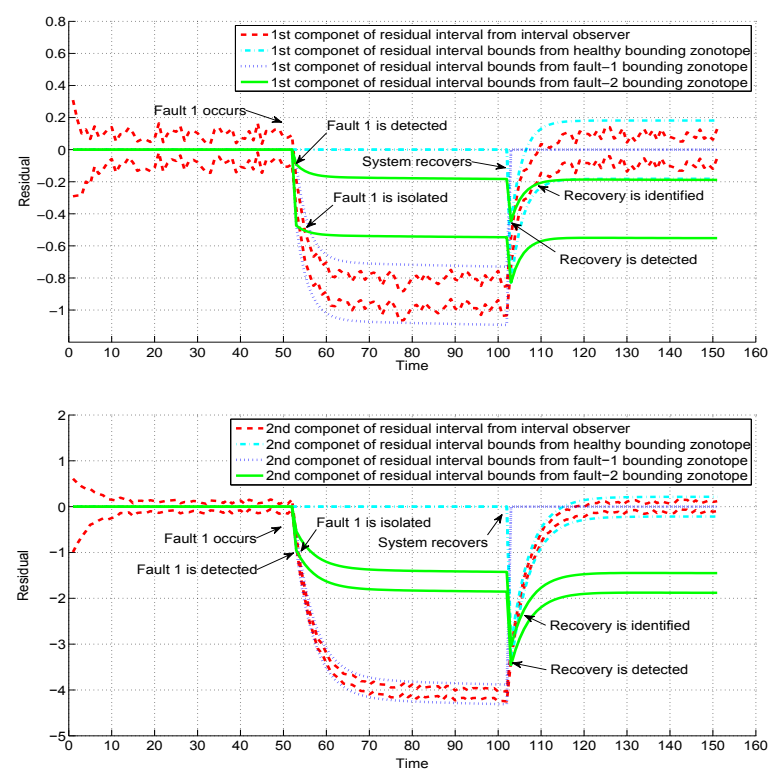

Fig. 1. FDI of the fault 1
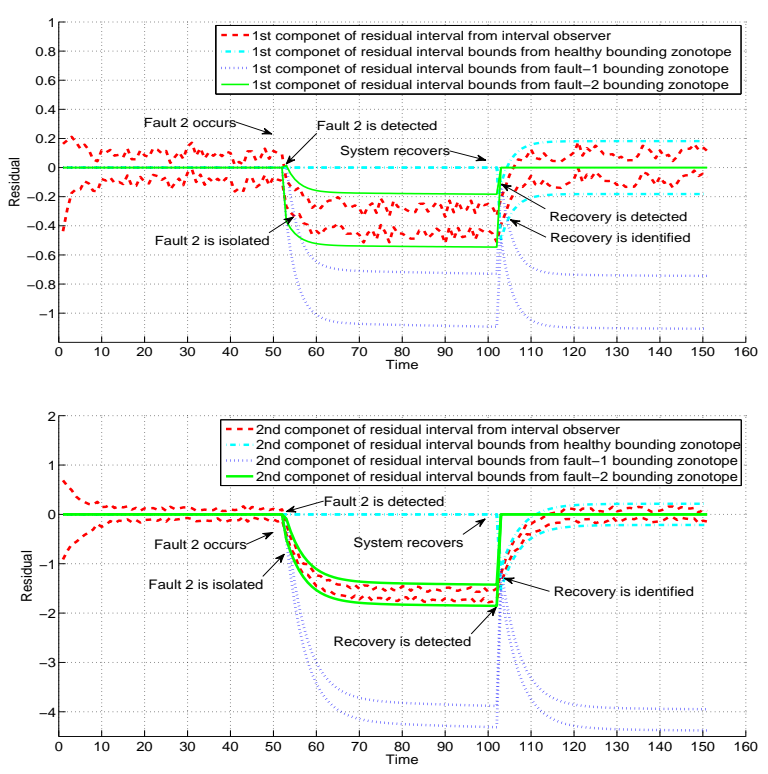

Fig. 2. FDI of the fault 2

\section{CONCLUSIONS}

In this paper, an interval observer-based FDI approach is proposed and a group of guaranteed FDI conditions based on invariant sets are established. An advantage of this approach is that it can isolate faults during the transition between different modes. The future research will focus on developing easier ways to construct initial zonotopes for residual-bounding zonotopes and exploring possibilities to further reduce the FDI conditions.

\section{ACKNOWLEDGEMENTS}

The work of F. Xu, V. Puig and C. Ocampo-Martinez has been supported by Spanish research project WATMAN (DPI2009-13744) of the Science and Technology Ministry, by the DGR of Generalitat de Catalunya (SAC group Ref. 2009/SGR/1491), by European Comission through contract i-Sense (FP7-ICT-2009-6-270428), and by China Scholarship Council (File No.2011629170). Besides, acknowledge Juan Manuel Grosso for significant discussions.

\section{REFERENCES}

[1] T. Alamo, J.M. Bravo, and E.F. Camacho. Guaranteed state estimation by zonotopes. In Decision and Control, 2003. Proceedings. 42nd IEEE Conference on, Maui, Hawaii, USA, December 2003.

[2] C. Combastel. A state bounding observer based on zonotopes. In European Control Conference, Cambridge, UK, 2003.

[3] P. Guerra, V. Puig, and M. Witczak. Robust fault detection with unknown-input interval observers using zonotopes. In Proceedings of the 17th World Congress, The International Federation of Automatic Control, COEX, Seoul, South Korea, July 2008.

[4] S. Olaru, J.A. De Doná, M.M. Seron, and F. Stoican. Positive invariant sets for fault tolerant multisensor control schemes. International Journal of Control, 83(12):2622-2640, 2010.

[5] R.J. Patton and J. Chen. Observer-based fault detection and isolation: Robustness and applications. Control Engineering Practice, 5(5):671 682, 1997. 